Published by Al-Nahrain College of Medicine P-ISSN 1681-6579

E-ISSN 2224-4719

Email: iraqijms@colmed-alnahrain.edu.iq http://www.colmed-alnahrain.edu.iq http://www.iraqijms.net

\title{
Human Cytomegalovirus Infection Among Neonates with Symptomatic Congenital Infections and Birth Defects
}

\author{
Sevan N. Alwan ${ }^{1} M S C$, Hala S. Arif ${ }^{2}$ CAMP, Atheer J. Al-Saffar ${ }^{3}$ FICMS, Haider S. Kadhim ${ }^{4} P h D$, \\ Brian L. Wickes ${ }^{5} P h D$, Jianmin $\mathrm{Fu}^{5} P h D$
}

\footnotetext{
${ }^{1}$ Dept. of Microbiology, College of Medicine, Baghdad University, Baghdad, Iraq, ${ }^{2}$ Dept. of Pediatrics, ${ }^{3}$ Dept. of Community and Family Medicine, ${ }^{4}$ Dept. of Microbiology, College of Medicine, Al-Nahrain University, Baghdad, Iraq, ${ }^{5}$ Advanced Nucleic Acid Core Facility, Dept. of Microbiology and Immunology, Medical School, University of Texas Health Science Center at San Antonio, Texas, United State of America.
}

\begin{abstract}
Background Human cytomegalovirus (HCMV) is the major viral etiology of congenital infection and birth defects, during current maternal infection the fetal transmission is high (30-40\%) and the symptomatic neonates have diseases involving the neurologic, hematopoietic, respiratory and other organ systems, causing high mortality and long-term sequelae.

Objective To measure the frequency of congenital and perinatal HCMV infection among symptomatic neonates and its possible burden of disease among them.

Methods A total of one hundred ninety-eight symptomatic neonates with clinical manifestations of overt congenital infection enrolled in this study from September 2014 to March 2015. Serum samples were obtained from each subject targeted in this study. HCMV infection was defined as HCMV-IgM antibody positive by Electrochemiluminescence Immunoassay (ECLIA) techniques.

Results The prevalence of HCMV infection among symptomatic neonates with congenital infection was 25 (12.6\%). The average age of HCMV detection was 9.96 (SD 6.73) days with a median of 7 days, a minimum of 3 days and a maximum of 28 days. Jaundice was the most predominant clinical finding $14(56 \%)$, followed in order of frequency by hepatomegaly $9(36 \%)$ and pneumonitis $7(28 \%)$.

Conclusion The high prevalence of neonatal HCMV infection among neonates with symptomatic congenital infections could indicate a high rate of maternal HCMV primary or current infection among our population.

Keywords HCMV, congenital infection, neonates, clinical finding.

DOI: 10.22578/IJMS.14.4.14
\end{abstract}

List of abbreviation: HCMV = Human Cytomegalovirus, ECLIA = Electrochemiluminescence Immunoassay.

\section{Introduction \\ $\mathrm{H}$ uman cytomegalovirus (HCMV) is the major viral etiology of congenital infection and birth defects ${ }^{(1)}$. \\ Congenital infection is the leading cause of $10 \%$ of neonatal deaths in Iraq (2). HCMV transmission rates to the fetus are significantly higher than Rubella and Toxoplasma gonidii ${ }^{(3)}$. During maternal primary or current (reinfection}

or reactivation) infection HCMV can translocate the placental barrier and can cause infection of the developing fetus ${ }^{(4)}$. Perinatal HCMV infection may be acquired from the mother as a result of contact with infected genital secretions during passage through the birth canal or via ingestion of the infected breast milk (perinatal infection). This postnatally acquired infection rarely results in significant symptoms or sequelae in full-term infants (5), the exception being the low-birth weight and 
prematurity. Infants born at preterm birth may be at higher risk of developing HCMV associated diseases ${ }^{(6,7)}$.

HCMV is a common congenital infection worldwide. The prevalence of congenital HCMV is $0.64 \%$ in developed countries (8) while reliable estimate of the prevalence of congenital HCMV infection in developing countries are not available ${ }^{(8,9)}$. Since the incidence of congenital infection is directly correlated with the seroprevalence of HCMV antibodies in the population ${ }^{(10,11)}$, congenital HCMV infection may indeed exert its greatest burden on developing countries with high birth rates and high seroprevalence, up to $95-100 \%$ (12).

Approximately $90 \%$ of newborns with congenital HCMV infection have no clinical symptoms of disease at birth, while about $10 \%$ of them have the signs and symptoms at birth ${ }^{(13,14)}$. Greater than $90 \%$ of symptomatic infants develop long-term neurological sequelae, such as sensorineural hearing loss, developmental disability, cerebral palsy, and impaired vision ${ }^{(15)}$. This incidence is far greater than that of the better-known chromosomal disorder Down syndrome ${ }^{(16)}$. On the other hand, among the $90 \%$ asymptomatic congenital HCMV infections, approximately $10-15 \%$ will later develop long-term neurological sequelae ${ }^{(15,17)}$. Hearing loss is the most common sequels of children with asymptomatic congenital HCMV infection who develop long-term sequelae $(17,18)$. Perinatal acquisition of HCMV has little significance in full-term infants and it is not associated with long-term disability. Prematurely born infants with postnatally HCMV were found to be at higher risk for HCMV associated diseases ${ }^{(6)}$. The factors that enhance the magnitude of HCMV as a health problem can be explained by the following factors: no specific antiviral therapy for HCMV infection and no licensed vaccine ${ }^{(19-21)}$, a lack of awareness of congenital HCMV among health care workers and the public because most maternal and newborn infections are asymptomatic and, therefore, are not recognized at birth ${ }^{(22)}$.

This study aimed to estimate the frequency rate of HCMV infection among symptomatic neonates with congenital infections and the possible burden of disease among the infected neonates.

\section{Methods}

A cross sectional study was conducted on neonates with congenital infection. A total of one hundred ninety-eight (198) neonates diagnosed with congenital infection were selected based on the inclusion criteria of the study from September 2014 to March 2015. All the selected neonates were admitted to the Neonatal Intensive Care Unit. Serum samples were obtained from the neonates, their ages ranged between one and thirty days. The neonates enrolled in this study were symptomatic neonates diagnosed with congenital infection the inclusion criteria as follows: various clinical manifestation like jaundice, petechial rash, hepatosplenomegaly, pneumonitis, congenital heart diseases (CHD), congenital malformations especially those involving the central nervous system and ophthalmological abnormalities (23). The exclusion criteria as follows: neonates were not considered suggestive if the only finding was prematurity ${ }^{(24)}$. Clinical manifestation was determined by consultation of a pediatric specialist and verification of the information in the medical record. Neonates were admitted to the Neonatal Intensive Care Unit in Child Protection Teaching Hospital and Imamein Kadhimein Medical City in Baghdad. About 1- 2 milliliters of venous blood was obtained from each subject targeted in this study. The blood samples were allowed to clot at room temperature and then centrifuged for serum. All sera were stored at $\left(-20^{\circ} \mathrm{C}\right)$ until testing. HCMV infection was defined as HCMV-IgM antibody positive by ECLIA Kits (Roche, Germany) according to the manufacture's protocol. 
Ethical approval to perform the study was obtained from the Research Ethical Committee at College of Medicine, Al-Nahrain University. Statistical analysis was performed in SPSS 24 using frequency analysis to calculate rate ratios, mean, median, maximum and minimum of the study variables.

\section{Results}

HCMV infection as indicated by specific IgM measured by Electrochemiluminescence Immunoassay (ECLIA) was detected in 25 out of $198(12.6 \%)$ symptomatic neonates as shown in figure (1).

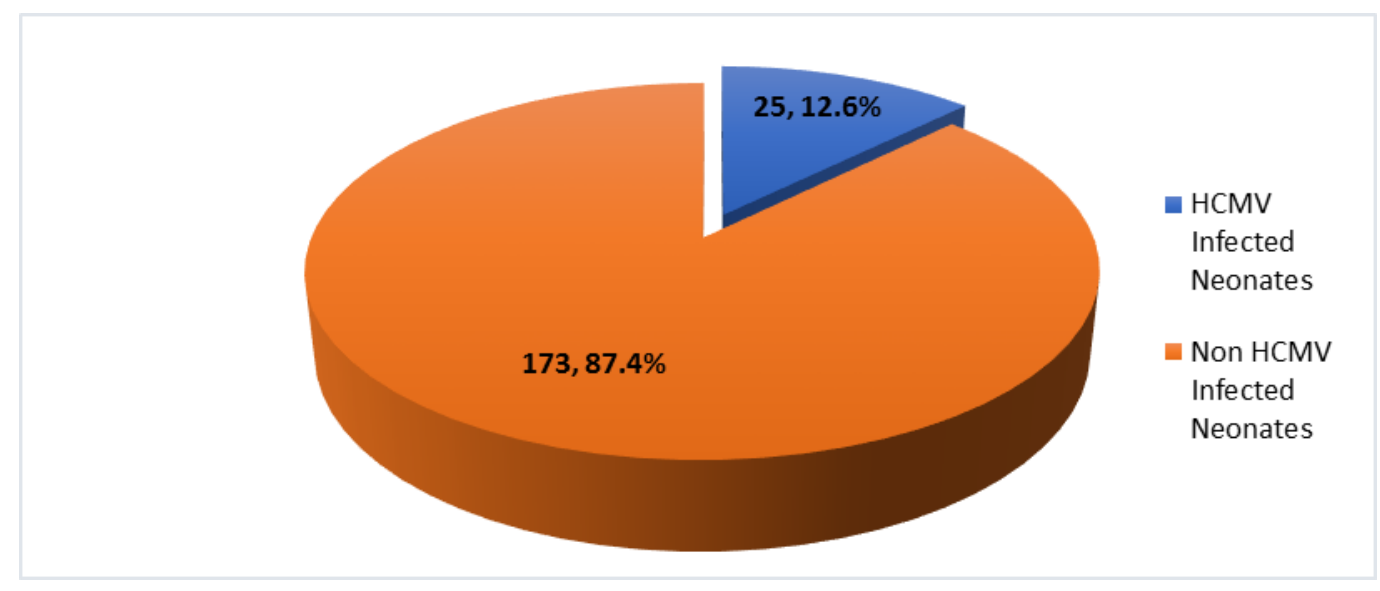

Figure 1. Positive IgM-Anti HCMV results among symptomatic neonates

Lower female rates 11 out of 25 (44\%) than males 14 out of 25 (56\%) were found among infected neonates. The average age of HCMV detection among symptomatic neonates was 9.96 days after birth. First week of the postpartum period was the medium age of HCMV detection. HCMV infection was detected among symptomatic neonates in a minimum age of 3 days and in a maximum age of 28 days after birth (SD 6.73) as shown in table (1).

Table 1. Characteristics of symptomatic neonates with HCMV infection

\begin{tabular}{lcccccc}
\hline \multicolumn{1}{c}{ Characteristics } & Number & Percentage & Mean & Median & Minimum & Maximum \\
\hline Infected neonates & 25 & 12.6 & & & & \\
\hline Sex & & & & & & \\
Female & 11 & 44 & & & & \\
Male & 14 & 56 & & & & 28 \\
\hline Age by days & & & 9.96 & 7 & 3 \\
\hline
\end{tabular}

Among the clinical manifestations of symptomatic neonates with serological evidence of congenital and perinatal HCMV infection, jaundice was the most predominant clinical finding 14 out of 25 (56\%), followed in order of frequency by hepatomegaly 9 out of 25 (36\%) pneumonitis 7 out of 25 (28\%), prematurity 6 out of 25 (24\%). Microcephaly was the most predominant neurological manifestation 6 out of 25 (24\%), followed in order of frequency by hydrocephaly 5 out of 25 (20\%), convulsion 2 out of $25(8 \%)$, and vision problems 1 out of 25 (4\%). Other clinical findings are shown in figure (2). 


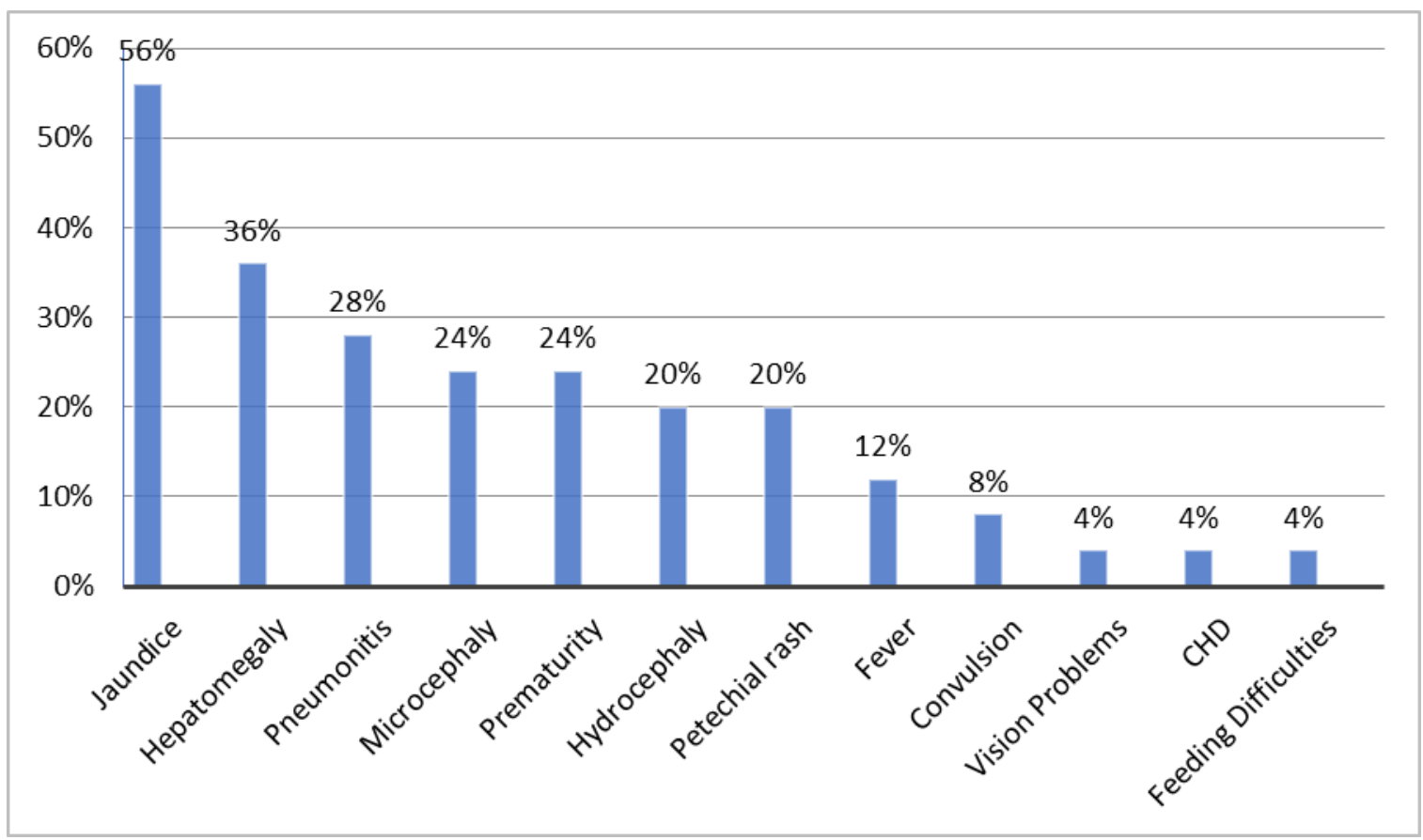

Figure 2. Clinical signs among symptomatic infected neonates, listed in order of frequency

\section{Discussion}

Among hospitalized neonates who were suggestive for congenital and perinatal HCMV infection, IgM-anti HCMV was detected in 25 (12.6\%) of them. A lower result of $6 \%$ was recorded among symptomatic neonates in Iraq previously by Al-Ali and his coworkers in 1995, though they conducted their work on cord sera only, so their results reflected the congenital HCMV infection only ${ }^{(25)}$. Approximately similar result was reported by the same author among suggestive live-born infants in Mosul, Iraq by using specific IgM as a screening test ${ }^{(11)}$. A higher finding was reported $16.1 \%$ in Baghdad, Iraq by Habib et al. in 2000 , by measuring specific IgM among symptomatic infants ${ }^{(24)}$. The higher results might be related to the wider range of age of suggestive infants included in that study as compared to this study. Higher results $20 \%$ of HCMV infection as indicated by specific IgM among symptomatic neonates was found in India ${ }^{(26)}$, while lower results $1.6 \%$ and $2 \%$ were reported among symptomatic infants in Iran and Palestine respectively using the same test ${ }^{(27,28)}$. The variation among these results and result of this study may reflect the variation of HCMV prevalence in different countries ${ }^{(29)}$. The variation of congenital HCMV epidemiology could be related to the maternal seropositivity because women of childbearing age, who are HCMV seronegative are at major risk of giving birth to infants with symptomatic congenital infection if primary infection is acquired during pregnancy ${ }^{(11)}$.

The median of HCMV detection was the first week of the postpartum period, a result that may indicate that most of the HCMV infection among symptomatic neonates was congenital infection rather than perinatal infection ${ }^{(30)}$.

Jaundice was the most frequently noted presenting sign 56\%, followed by hepatomegaly $36 \%$. These results are comparable to those documented in Iraq regarding the most frequent signs and also the order of frequency among symptomatic HCMV infected infants, Habib et al. reported $65.2 \%$ for jaundice followed by $41.1 \%$ for hepatomegaly (24). A similar finding was reported among hospitalized HCMV infected children in Palestine, but hepatomegaly was the first sing in order of frequency $60 \%$ 
followed by jaundice $30 \%{ }^{(28)}$. The variation in the order of frequency of jaundice and hepatomegaly between hospitalized symptomatic HCMV infected children in that study and the present study may be related to the variation in HCMV strains. There is evidence indicating that the existence of HCMV variant plays an important role in the pathogenesis of disease ${ }^{(31)}$. Jaundice was reported in $63 \%$ of symptomatic HCMV infected infants by Pass and his colleagues ${ }^{(32)}$, and $70 \%$ in another study ${ }^{(33)}$. In the presented study hepatomegaly was comparable to that documented by others ${ }^{(34,35)}$. However, higher figures $74 \%$ and $70 \%$ were reported by other workers ${ }^{(32,33)}$.

Pneumonitis was found in $28 \%$ of symptomatic HCMV infected neonates, a result which is found to be higher than that recorded in a study from Iraq $11.6 \%$ among infected infants (24). In a recent study, a higher frequency of pneumonitis was detected in $47 \%$ of symptomatic infected children (36). The variation in the results may be due to the fact that the clinical diagnosis of HCMV lung infection is challenging in children and often requires a high-index of suspicion ${ }^{(37)}$.

Prematurity was found in $24 \%$ of symptomatic infected. Meier et al. have reported significant risk of HCMV transmission postnatally in preterm infants with the possibility of severe disease ${ }^{(43)}$. Immature immune system in infants, as a consequence of prematurity among neonates relates to lower production of cytokines which reduces the ability of T-cell activation and viral detection as compared to term infants ${ }^{(44)}$. In addition, premature infants are at high risk for symptomatic HCMV infection as they don't receive as many as transplacental HCMV antibodies, and the antibodies that they do receive disappear more rapidly through catabolism than in infants born near term ${ }^{(45,46)}$.

Interestingly, result in the current study is higher than the results reported in a study in Iraq, which indicated $16.7 \%$ prematurity among symptomatic HCMV infected infants even though the age range in that study was wider than current study (24). This finding could indicate that prematurity is more likely to correlate with congenital infection since HCMV impairs placental development and functions regardless of virus transmission to fetus, leading to intrauterine growth restriction that results in the baby being preterm ${ }^{(10)}$. In addition, HCMV efficiently spreads into fetal organs following fetal viremia during HCMV congenital infection. The major target organ is the fetal lungs. HCMV replication in the lungs triggers apoptosis near and within viral lesions and impairs the production of surface proteins of the lung that adversely impact lung development (47). However, further studies need to be conducted on premature neonates with congenital and perinatal HCMV infections separately to evaluate the association between prematurity and each type of neonatal HCMV infection.

The most frequent neurological manifestations among symptomatic infected children were microcephaly and hydrocephaly, recorded at $24 \%$ and $20 \%$ respectively. A higher result was reported for microcephaly $38.9 \%$, and an approximately similar result for hydrocephaly in Iraq among symptomatic infected infants by Habib et al. ${ }^{(24)}$. Other study in Iraq done by AlAli et al. described hydrocephaly in $33.3 \%$ of infected newborns and has also shown hydrocephaly as the most frequent neurological clinical finding (25). In contrast, microcephaly and hydrocephaly were not recorded among hospitalized children with symptomatic HCMV infection in Palestine ${ }^{(28)}$. Gandhoke et al. have reported an equal frequency of microcephaly and hydrocephaly among symptomatic infected children (26). Other neurological manifestations recorded were convulsion $8 \%$ and vision problems $4 \%$. In two studies conducted in Iraq among symptomatic HCMV infected neonates and infants, convulsion and vision problems were recorded in lower frequency compared to this 
study ${ }^{(24,25)}$. The variation in the results may be related to the wider age range of these two studies compared with the present study. A systemic review of 30 studies regarding hearing problems revealed that hearing loss was $12.65 \%$ among symptomatic HCMV infected children ${ }^{(38)}$. In the present study, hearing problems was not detected among neurological manifestations in symptomatic HCMV infected neonates. A large number of hearing problems that result from HCMV infection was detected at school age ${ }^{(39)}$, which may be attributed to misdiagnosis of hearing problems among neonates in this study.

Petechial rash was described in $20 \%$ of the symptomatic HCMV infected neonates, which was found to be lower than those recorded by others: $33.3 \%$ and $32 \%{ }^{(40)}$. In contrast, petechial rash was not recorded by Gandhoke ${ }^{(26)}$ and Neirukh et al. ${ }^{(28)}$. This variation in the results may be due to the possibility that this clinical sign might have passed unnoticed by the physician as it may be transient, disappearing with 48 hours ${ }^{(41)}$ and requires very careful clinical examination.

Fever for unknown reasons was shown in $12 \%$ of symptomatic HCMV infected neonates. Fever for unknown reasons may indicate the prenatal HCMV infection among neonates in this study since it presents the usual clinical picture of HCMV mononucleosis among immunocompetent children ${ }^{(42)}$. Heart diseases and feeding difficulties were last regarding the order of frequency. The variations in the results of clinical findings that were found among different studies may reflect the variations in the severity of disease and the degree of organ involvement. Clinical findings associated with HCMV infection are broad and non-specific ${ }^{(13)}$. This variation may be related to the type of HCMV genotype and tissue tropism associated with the virus strain. From a prognostic viewpoint, the prompt identification of infected infants would help define a population at risk for developmental abnormalities so the establishment of diagnosis early in life is important for the infants and for the families.
Acknowledgement:

We would like to thank all patients and their families for their acceptance to participate in this study. Our grateful to staff of Child Protection Teaching Hospital and Pediatric Department in Imamein Kadhimein Medical City in Bagdad for their help in samples collections and laboratory work.

\section{Author Contribution}

Alwan: collection of the sample, conduction the experimental aspects of the study, and writing the manuscript. Kadhim: concepts of the study, revision and approval the final version of the manuscript. Al-Saffar: the study design, implementation of the statistical aspects, revision of the final version of the manuscript. Arif: clinical consultation. Wickes: general consultation. Fu: experimental consultation.

\section{Conflict of Interest}

Authors declare no conflict of interest.

\section{Funding}

Self-funding.

\section{References}

1. El-Sayed MS, Goldfarb DM, Fulford M, et al. Severe late-onset multisystem cytomegalovirus infection in a premature neonate previously treated for congenital infection. BMC Pediatrics. 2013; 13: 142. doi:10.1186/1471-2431-13-142.

2. Awqati NA, Ali MM, Al-Ward NJ. Causes and differentials of childhood mortality in Iraq. BMC Pediatrics. 2009, 9: 40. doi:10.1186/1471-2431-9-40.

3. Margioula-Siarkon C, Kalogiannidis I, Petousis S, et al. Cytomegalovirus, Toxoplasma gonidi and Rubella vertical transmission rates according to mid. trimisters amniocentesis: A Retrospective study. Int J Prev Med. 2015; 6: 32 . doi:10.4103/20087802.154774

4. Sykes L, Maclntyre DA, Yap XJ, et al. Changes in the Th1:Th2 cytokine bias in pregnancy and the effects of the anti-inflammatory cyclopentenone prostaglandin 15-deoxy-Delta12,14-prostaglandin J2. Mediat Inflam. 2012; 2012. doi: 10.1155/ 2012/ 416739.

5. Schleiss MR. Acquisition of human cytomegalovirus infection in infants via breast milk: natural immunization or cause for concern? Rev Med Virol. 2006; 16(2): 73-82. 
6. Buxmann $H$, Miljak A, Fischer $D$, et al. Incidence and clinical outcome of cytomegalovirus transmission via breast milk in preterm infants $\leq 31$ weeks. Acta Paediatrica. 2009; 98(2): 270-6.

7. Kurath S, Halwachs-Baumann G, Muller W, et al. Transmission of cytomegalovirus via breast milk to the prematurely born infant: a systematic review. Clinical Microbiology and Infection. 2010; 16(8): 1172-8.

8. Griffiths $P$, Plotkin S, Mocarski E, et al. Desirability and feasibility of a vaccine against cytomegalovirus. vaccine, 2013; 31S: B197-B203.

9. Dollard SC, Grosse SD, Ross DS. New estimates of the prevalence of neurological and sensory sequelae and mortality associated with congenital cytomegalovirus infection. Rev Med Virol. 2007; 17(5): 355-63.

10. Lenore $P$, Matthew $P$, Alex F. Intaruterine growth restriction by underlying congenital cytomegalovirus infection. J Infect Dis. 2014; 209(10): 1573-84.

11. Kenneson A, Cannon MJ. Review and meta-analysis of the epidemiology of congenital cytomegalovirus (CMV) infection. Rev Med Virol. 2007; 17(4): 253-76.

12. Mocarski ES, Shenk T, Pass RF. Cytomegaloviruses: fields virology. 5th ed. Philadelphia: Lippincott Williams; 2007. p. 2701-72.

13. Swanson EC, Schleiss MR. Congenital cytomegalovirus infection: new prospects for prevention and therapy. Pediatr Clin North Am. 2013; 60(2): 335-49.

14. Kylat RI, Kelly EN, Ford-Jones EL. Clinical Finding and adverse outcome in neonates with symptomatic congenital cytomegalovirus (SCCMV) infection. Eur J Pediatr. 2006; 165: 773-8.

15. Coll O, Benoist G, Ville $Y$, et al. Guidelines on CMV congenital infection. J Perinat Med. 2009; 37: 433-45.

16. Demmler-Harrison GJ. Congenital cytomegalovirus: public health action towards awareness, prevention, and treatment. J Clin Virol. 2009; 46(4): S1-S5.

17. Krakar G, Dakovic I, Dellin S, et al. Evolutive leukoencephalopathy in congenital cytomegalovirus infection. J Child Neurol. 2015; 30(1); 93-195.

18. Smiechura M, Struzycka M, Konopka W. Congenital cytomegalovirus infection and hearing evaluation in Children. Otolaryngologia Polska. 2014; (68): 6: 3037.

19. Lombardi G, Garofoli F, Stronati M. Congenital cytomegalovirus infection: treatment, sequelae and follow-up. J Matern Fetal Neonatal Med. 2010; 23(3): 45-8.

20. Dasari V, Smith C, Khanna R. Recent advances in designing an effective vaccine to prevent cytomegalovirus-associated clinical diseases. Expert Rev Vaccines. 2013;12(6): 661-76. doi: 10.1586/erv.13.46.
21. Shedlock DJ, Talbott KT, Wu SJ. Vaccination with synthetic construct expressing cytomegalovirus immunogens in highly T-cell immunogenic in mice. Human Vaccines Immunotherap. 2012; 8(11): 166881.

22. Manickals S, Emery VC, Lazzorotto T. et al. The "Silent" global burden of congenital cytomegalovirus. Clin Microbiol Rev. 2013; 26(1): 86-102.

23. Goderis J, De Leenheer E, Smets K, et al. Hearing Loss and Congenital CMV Infection: A Systematic Review. Pediatrics. 2014; 134(5): 912-82.

24. Habib MA, Al-Omar LS, Sameh H. Prevalence of HCMV infection among Iraqi infants. Iraqi J Med Sci. 2003; 2: 76-82.

25. Al Ali HY, Yasseen SA, Al-Rawi S. congenital CMV infection among newborn infants with congenital malformation in Mosul, Iraq. Jordan Med J. 1995; 32: 60-4.

26. Gandhoke G, Hussain SA, Pasha ST, et al. Glycoprotein B genotyping in congenital/ perinatal cytomegalovirus infection in symptomatic infants. India Pediatrics. 2013; 50: 663-7.

27. Golalipour MJ, Khodabakhshi B, Ghaemi E. Possible role of TORCH agents in congenital malformations in Gorgan Northern Islamic Republic of Iran. Eastern Mediter Health J. 2009; 15(2): 330-6.

28. Neirukh T, Qaisi A, Saleh N, et al. Seroprevalence of Cytomegalovirus among pregnant women and hospitalized children in Palestine. BMC Infect Dis. 2013; 13: 528. doi: 10.1186/1471-2334-13-528.

29. Cannon MJ, Schmid DS, Hyde TB. Review of cytomegalovirus seroprevalence and demographic characteristics associated with infection. Rev Med Virol. 2010; 20(4): 202-13.

30. Arellano-Galindo J, Villanueva-Garcia D, Cruz-Ramirez $\mathrm{JL}$, et al. Detection and genotyping of CMV in Mexican preterm infants in the context of maternal seropositivity. J Infect Dev Ctries. 2014; 8(6): 758-67.

31. de Vries JJ, Vesseur A, Rotteveel LJ, et al. Cytomegalovirus DNA detection in dried blood spots and perilymphatic fluids from pediatric and adult cochlear implant recipients with prelingual deafness. J Clin Virol. 2013; 56: 113-7.

32. Pass RF, Stagno S. Outcome of systemic congenital CMV infection. Paediatrics. 1980; 66: 758-62.

33. Boppana SB, Pass RF. Symptomatic congenital CMV infection and neonatal morbidity and mortality. Pediatr Infec Dis J. 1992; 11: 93-9.

34. Broor S, Kapil A, Kishore J, et al. Prevalence of rubella virus and $\mathrm{CMV}$ infection in suspected cases of Congenital infection. Indian J Pediatr. 1991; 58(1): 75-8.

35. Dobbins JG, Stewart JA, Demnler GJ. Surveillance of congenial CMV disease, 1990-1991 collaborating 
registry group. MMWR-CDC-Surveill-Summ. 1992. 41(2): 35-9.

36. Restrepo-Gualteros SM, Jaramillo LE, Gonzalez-Santo $M$, et al. Characterization of Cytomegalovirus Lung Infection in Non-HIV Infected Children. Viruses. 2014; 6: 2038-51.

37. Radigan KA, Wunderink RG. Epidemic viral pneumonia and other emerging pathogens. Clin Chest Med. 2011; 32: 451-67.

38. Goderis J, De Leenheer E, Smets $K$, et al. Hearing Loss and congenital CMV infection: A systematic review. Pediatrics. 2014; 134 (5): 912-82.

39. Gabrielli1 L, Bonasoni MP, Santini D. Congenital cytomegalovirus infection: patterns of fetal brain damage. Clin Microbiol Inf. 2012; 18: E419-E426.

40. Dreher AM, Arora N, Fowler KB, et al. Spectrum of Disease and Outcome in Children with Symptomatic Congenital Cytomegalovirus Infection. J Pediatr. 2014; 164(4): 855-9.

41. Hanshow JB, Dudgeon JA, Marshal WC. Congenital CMV. In: Hanshaw JB. Viral disease of the fetus and newborn. $2^{\text {nd }}$ ed. Philadelphia: WB Saunders; 1985. p. 29-131.

42. Rodriguez-Bano J, Muniain MA, Borobio MV. Cytomegalovirus mononucleosis as a cause of prolonged fever and prominent weight loss in immunocompetent adults. Clin Microbiol Infect. 2004; 10(5): 468-70.
43. Meier J, Lienecke U, Tschirch E, et al. Human cytomegalovirus reactivation during lactation and mother-to-child transmission in preterm infants. J Clin Microbiol. 2005; 43(3): 1318-24.

44. Melville JM, Moss TJ. The immune consequences of preterm birth. Neuroscience. 2013; 7(79). doi: 10.3389/finins. 2013. 00079.

45. Lanzieri TM, Dollard SC, Bialek SR et al. Systematic review of the birth prevalence of congenital cytomegalovirus infection in developing countries. Int J Infect Dis. 2014; 22: 44-8.

46. Romero-Gomez MP, Cabrena M, Montes-Bueno MT, et al. Evaluation of cytomegalovirus infection in low birth weight children by breast milk using a real-time polymerase chain reaction assay. J Med Virol. 2015; 87; 845-50.

47. Maidji E, Kosikova G, Joshi $P$, et al. Impaired surfactant production by alveolar epithelial cells in SCID-hu lung mouse model of congenital cytomegalovirus infection. J Virol. 2012; 86(23): 12795-805.

\section{Correspondence to Sevan N. Alwan}

E-mail: sevan.samer@gmail.com Received 15 ${ }^{\text {th }}$ Aug. 2016: Accepted $30^{\text {th }}$ Nov. 2016 